\title{
Aerodynamic Shape Optimization of a Vertical-Axis Wind Turbine Using Differential Evolution
}

\author{
Travis J. Carrigan, Brian H. Dennis, Zhen X. Han, and Bo P. Wang \\ Department of Mechanical and Aerospace Engineering, The University of Texas at Arlington, P.O. Box 19023, \\ Arlington, TX 76019-0023, USA \\ Correspondence should be addressed to Brian H. Dennis, dennisb@uta.edu
}

Received 1 September 2011; Accepted 3 October 2011

Academic Editors: S. S. Kalligeros and S. Senthilarasu

Copyright ( $(2012$ Travis J. Carrigan et al. This is an open access article distributed under the Creative Commons Attribution License, which permits unrestricted use, distribution, and reproduction in any medium, provided the original work is properly cited.

\begin{abstract}
The purpose of this study is to introduce and demonstrate a fully automated process for optimizing the airfoil cross-section of a vertical-axis wind turbine (VAWT). The objective is to maximize the torque while enforcing typical wind turbine design constraints such as tip speed ratio, solidity, and blade profile. By fixing the tip speed ratio of the wind turbine, there exists an airfoil crosssection and solidity for which the torque can be maximized, requiring the development of an iterative design system. The design system required to maximize torque incorporates rapid geometry generation and automated hybrid mesh generation tools with viscous, unsteady computational fluid dynamics (CFD) simulation software. The flexibility and automation of the modular design and simulation system allows for it to easily be coupled with a parallel differential evolution algorithm used to obtain an optimized blade design that maximizes the efficiency of the wind turbine.
\end{abstract}

\section{Introduction}

1.1. Alternative Energy. As the world continues to use up nonrenewable energy resources, wind energy will continue to gain popularity. A new market in wind energy technology has emerged that has the means of efficiently transforming the energy available in the wind to a usable form of energy, such as electricity. The cornerstone of this new technology is the wind turbine.

A wind turbine is a type of turbomachine that transfers fluid energy to mechanical energy through the use of blades and a shaft and converts that form of energy to electricity through the use of a generator. Depending on whether the flow is parallel to the axis of rotation (axial flow) or perpendicular (radial flow) determines the classification of the wind turbine.

1.2. Wind Turbine Types. Two major types of wind turbines exist based on their blade configuration and operation. The first type is the horizontal-axis wind turbine (HAWT). This type of wind turbine is the most common and can often be seen littered across the landscape in areas of relatively level terrain with predictable year round wind conditions. HAWTs sit atop a large tower and have a set of blades that rotate about an axis parallel to the flow direction. These wind turbines have been the main subject of wind turbine research for decades, mainly because they share common operation and dynamics with rotary aircraft.

The second major type of wind turbine is the vertical axis wind turbine (VAWT). This type of wind turbine rotates about an axis that is perpendicular to the oncoming flow, hence, it can take wind from any direction. VAWTs consist of two major types, the Darrieus rotor and Savonius rotor. The Darrieus wind turbine is a VAWT that rotates around a central axis due to the lift produced by the rotating airfoils, whereas a Savonius rotor rotates due to the drag created by its blades. There is also a new type of VAWT emerging in the wind power industry which is a mixture between the Darrieus and Savonius designs.

1.2.1. Vertical-Axis Wind Turbines. Recently, VAWTs have been gaining popularity due to interest in personal green energy solutions. Small companies all over the world have been marketing these new devices such as Helix Wind, 
Urban Green Energy, and Windspire. VAWTs target individual homes, farms, or small residential areas as a way of providing local and personal wind energy. This reduces the target individual's dependence on external energy resources and opens up a whole new market in alternative energy technology. Because VAWTs are small, quiet, easy to install, can take wind from any direction, and operate efficiently in turbulent wind conditions, a new area in wind turbine research has opened up to meet the demands of individuals willing to take control and invest in small wind energy technology.

The device itself is relatively simple. With the major moving component being the rotor, the more complex parts like the gearbox and generator are located at the base of the wind turbine. This makes installing a VAWT a painless undertaking and can be accomplished quickly. Manufacturing a VAWT is much simpler than a HAWT due to the constant cross-section blades. Because of the VAWTs simple manufacturing process and installation, they are perfectly suited for residential applications.

The VAWT rotor, comprised of a number of constant cross-section blades, is designed to achieve good aerodynamic qualities at various angles of attack. Unlike the HAWT where the blades exert a constant torque about the shaft as they rotate, a VAWT rotates perpendicular to the flow, causing the blades to produce an oscillation in the torque about the axis of rotation. This is due to the fact that the local angle of attack for each blade is a function of its azimuthal location. Because each blade has a different angle of attack at any point in time, the average torque is typically sought as the objective function. Even though the HAWT blades must be designed with varying cross-sections and twist, they only have to operate at a single angle of attack throughout an entire rotation. However, VAWT blades are designed such that they exhibit good aerodynamic performance throughout an entire rotation at the various angles of attack they experience leading to high time averaged torque. The blades of a Darrieus VAWT (D-VAWT) accomplish this through the generation of lift, while the Savonius-type VAWTs (S-VAWTs) produce torque through drag.

1.3. Computational Modeling. The majority of wind turbine research is focused on accurately predicting efficiency. Various computational models exist, each with their own strengths and weaknesses that attempt to accurately predict the performance of a wind turbine. Descriptions of the general set of equations that the methods solve can be found in Section 2. Being able to numerically predict wind turbine performance offers a tremendous benefit over classic experimental techniques, the major benefit being that computational studies are more economical than costly experiments.

A survey of aerodynamic models used for the prediction of VAWT performance was conducted by $[1,2]$. While other approaches have been published, the three major models include momentum models, vortex models, and computational fluid dynamics (CFD) models. Each of the three models are based on the simple idea of being able to determine the relative velocity and, in turn, the tangential force component of the individual blades at various azimuthal locations.

1.3.1. Computational Fluid Dynamics. Due to its flexibility, CFD has been gaining popularity for analyzing the complex, unsteady aerodynamics involved in the study of wind turbines $[3,4]$ and has demonstrated an ability to generate results that compare favorably with experimental data $[5$, 6]. Unlike other models, CFD has shown no problems predicting the performance of either high- or low-solidity wind turbines or for various tip speed ratios. However, it is important to note that predicting the performance of a wind turbine using CFD typically requires large computational domains with sliding interfaces and additional turbulence modeling to capture unsteady affects; therefore, CFD can be computationally expensive.

1.4. Objectives. The objective of the present work is to demonstrate a proof-of-concept optimization system and methodology similar to that which was introduced by [8], while aiming to maximize the torque, hence, the efficiency of a VAWT for a fixed tip speed ratio. To accomplish this, an appropriate model for predicting the performance of a VAWT is to be selected along with a robust optimization algorithm and flexible family of airfoil geometries.

Recent research has been conducted coupling models used for performance prediction with optimization algorithms. Authors in [9] used CFD coupled with a design of experiments/response surface method approach, focusing on only symmetric blade profiles in two dimensions using a seven-control-point Bezier curve. Bourguet only simulated one blade with a low solidity as to avoid undesirable unsteady effects. He found that when there exists a possibility of several local optima, stochastic optimization algorithms are better suited for the job as they tend to be more efficient than gradient-based algorithms. Authors of [10] and [11] coupled-low order performance prediction methods with optimization algorithms in a multicriteria optimization routine. Both of their approaches focused on HAWTs rather than VAWTs. Research has also led to patented blade designs using CFD coupled with optimization [12]. Other than using optimization techniques, inverse design methods can also be used to find an optimum design for a fixed tip speed ratio that satisfied the specified design performance characteristics. However, inverse design techniques require experience and intuition in order to specify desired performance, whereas optimization allows for designs to be generated that are more often than not beyond the intuition of a designer.

After reviewing the available models and recent research efforts, CFD was chosen as the appropriate tool for predicting the performance of a VAWT because of its flexibility and accuracy. Due to the possibility of local optima, and the requirement for floating-point optimization for geometric flexibility, a parallel stochastic differential evolution algorithm was chosen for the optimization. The NACA 4series family of wing sections was chosen as the geometry 
to be parameterized for the optimization, allowing either symmetric or cambered airfoil shapes to be generated. What separates this approach from all previous work is the consideration of both symmetric and cambered airfoil geometries, along with a full two-dimensional, unsteady simulation for a three-bladed wind turbine for various design points.

\section{Vertical Axis Wind Turbine Performance}

2.1. Wind Speed and Tip Speed Ratio. According to the National Climatic Data Center, the average annual wind speed in the United States is approximately $4 \mathrm{~m} / \mathrm{s}$ [13]. Realizing that the majority of wind turbines that have been developed to this day typically start producing power in winds as low as $3 \mathrm{~m} / \mathrm{s}$, the standard rated wind speed is still as high as $12 \mathrm{~m} / \mathrm{s}$. Determining the wind speed at which a wind turbine will operate is the most important step in predicting its performance and even aids in defining the initial size of the wind turbine. Once the operating wind speed of the turbine has been decided upon, the first step in wind turbine design is to select a operating tip speed ratio [14] which can be expressed by

$$
\lambda=\frac{\omega r}{V_{\infty}}
$$

or the ratio of the rotational velocity of the wind turbine $\omega r$ and the freestream velocity component (wind speed) $V_{\infty}$.

2.2. Geometry Definition. Once $\lambda$ has been chosen, the geometry of the VAWT can be defined through a dimensionless parameter known as the solidity

$$
\sigma=\frac{N c}{d}
$$

which is a function of the number of blades $N$, the chord length of the blades $c$, and the diameter of the rotor $d$. The solidity represents the fraction of the frontal swept area of the wind turbine that is covered by the blades.

2.3. Performance Prediction. With $\lambda$ chosen and the basic geometry of the VAWT defined, the next step is to predict the actual performance of the wind turbine. To do this, it is important to determine the forces acting on each blade. This is governed by the relative wind component $W$ and the angle of attack $\alpha$ seen in the snapshot of a D-VAWT blade cross-section in Figure 1. As the blade rotates, the local angle of attack $\alpha$ for that blade changes due to the variation of the relative velocity $W$. The induced velocity $V_{i}$ and the rotating velocity $\omega r$ of the blade govern the orientation and magnitude of the relative velocity. This in turn changes the lift $L$ and the $\operatorname{drag} D$ forces acting on the blade. As the lift and drag change both their magnitude and orientation, the resultant force $F_{R}$ changes. The resultant force can be decomposed into both a normal component $F_{N}$ and a tangential component $F_{T}$. It is this tangential force component that drives the rotation of the wind turbine and produces the torque necessary to generate electricity.

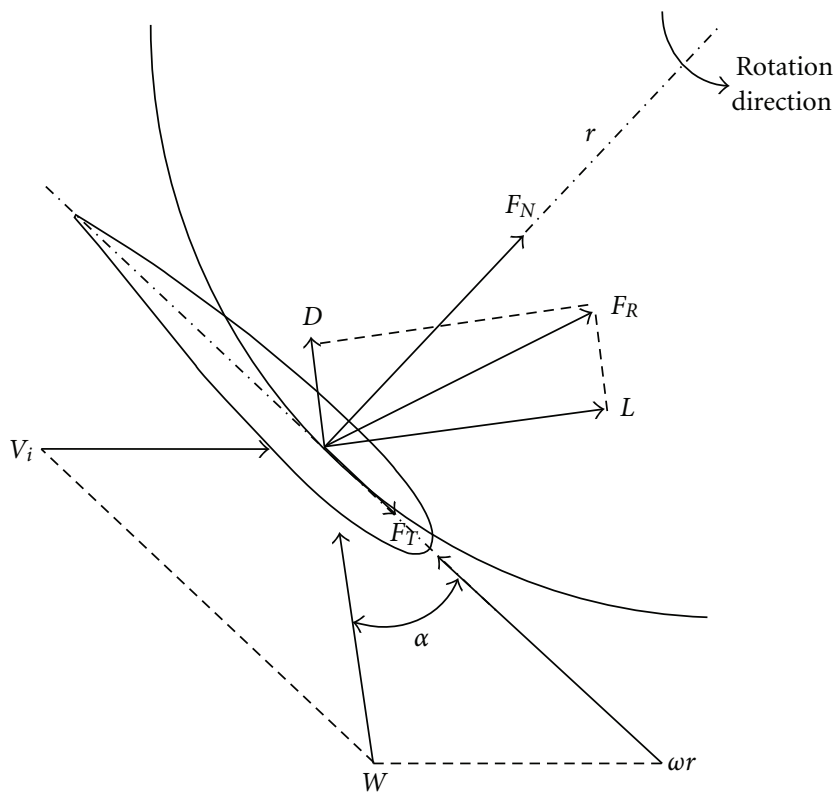

Figure 1: Velocity and force components for a Darrieus-type VAWT.

2.3.1. Average Torque. Close inspection of the underlying physics involved in wind turbine aerodynamics reveals that $\alpha$ is governed by the tip speed ratio $\lambda$ and, once determined, $L$ and $D$ can be found using empirical data or calculated using CFD. $L$ and $D$ are then nondimensionalized by dividing through by the dynamic pressure to obtain the coefficient of lift $C_{l}$ and the coefficient of drag $C_{d}$. These coefficients are used to calculate the tangential force coefficient

$$
C_{t}=C_{l} \sin \alpha-C_{d} \cos \alpha .
$$

To retrieve the actual tangential force, $C_{t}$ is multiplied by the dynamic pressure

$$
F_{T}=\frac{1}{2} C_{t} \rho \operatorname{ch} W^{2}
$$

where $\rho$ is the air density and $h$ is the height of the wind turbine. It is important to note that (4) represents the tangential force at only a single azimuthal position. Therefore, the process of determining $\alpha, C_{t}$, and $F_{T}$ must be repeated at all azimuthal locations before the torque can be calculated.

Because $F_{T}$ is calculated at all azimuthal locations, it is said to be a function of $\theta$ and the average tangential force for a single rotation of one blade is

$$
F_{\text {Tavg }}=\frac{1}{2 \pi} \int_{0}^{2 \pi} F_{T}(\theta) d \theta
$$

where the average torque for $N$ blades located at radius $r$ from the axis of rotation is given by

$$
\tau=N F_{T \text { avg }} r
$$


2.3.2. Power and Efficiency. The final step in predicting the performance of the wind turbine is determining the power it is able to extract from the wind and how efficiently it can accomplish that task. The amount of power the wind turbine is able to draw from the wind is given by

$$
P_{T}=\tau \omega
$$

Therefore, the efficiency of the wind turbine is simply the ratio of the power produced by the wind turbine and the power available in the wind given by the expression

$$
\mathrm{COP}=\frac{P_{T}}{P_{W}}=\frac{\tau \omega}{1 / 2 \rho d h V_{\infty}^{3}} .
$$

Equation (8) is significant in this work because it represents a nondimensional coefficient of performance (COP) that is a function of the torque to be used as the objective function for the aerodynamic shape optimization.

It should be mentioned that the goal in designing a wind turbine is to do so in such a way to extract as much energy as possible. Analyzing (5) and (8) reveals that, while an increase in the height of the wind turbine would increase $F_{T}$, hence $\tau$, theoretically COP would remain unaffected. However, if an increase in $P_{T}$ is all that is desired, the height of the wind turbine could be increased. In order to increase the efficiency of the wind turbine for a given $\lambda$, the blade shape and $\sigma$ would have to be adjusted. Equation (5) is a function of $C_{t}$ and $c$, where $C_{t}$ is a function of the blade shape and $c$ is a function of $\sigma$. Because the shape of the blade, $\sigma$, and $C_{t}$ are tightly coupled, it makes it difficult to select a geometry that maximizes the efficiency. Therefore, accomplishing this task is not straightforward and requires an iterative approach and the implementation of a simple, automated optimization methodology.

\section{Methodology}

3.1. Requirements. For the objectives of the current study to be feasible, the requirement for a straightforward, modular, and automated design framework became realized. Successfully taking a physical system, such as a wind turbine, and attempting to adjust, analyze, and optimize the design to satisfy an objective, or multiple objectives, required more than a few bundled files and programs requiring user input. In fact, it was this realization that sparked the idea of a simple, automated optimization methodology. The optimization methodology proposed is a unique and modular system aimed at relaxing the ties between the computer and operator and simplifying the design process so more time can be spent analyzing a solution and understanding the physics of the problem.

3.2. Unique Modular Design. A modular system is one in which entire parts of the system can be removed and replaced without compromising the process flows within the system. Therefore, for a module to be removed and replaced, it must be substituted with a module of equivalent functionality. Figure 2 illustrates the concept as it applies to wind turbine optimization and the methodology used in this work. The first step in the optimization process is generating the geometry. This geometry is described as a set of cartesian coordinates and is passed to the mesh generation module. This tool is used to discretize the fluid domain and output a specific file to the CFD solver module. This module calculates a solution and passes information to the postprocessing module. The postprocessing tools manipulate the data, calculate the objective function value, and pass it to the optimizer. If the objective function is considered a maximum value, the optimization terminates. If not, the process starts over. Details pertaining to each of the modules used in this work will be discussed in the next section.

\section{Toolbox}

4.1. Geometry Generation. The objective of the optimization was to find an aerodynamic shape that for a fixed tip speed ratio would maximize the efficiency of the wind turbine. The first step was to select a suitable shape, or series of shapes, that could be adjusted throughout the optimization process. An obvious choice was the NACA 4-series airfoil. The majority of VAWTs utilize NACA airfoil sections because they are easy to manufacture and their characteristics are widely available.

4.1.1. NACA 4-Series Airfoils. The NACA 4-series airfoil sections are defined by a mean camber line and a thickness distribution. In Figure 3, the mean camber line is the dashed line that splits the airfoil in half. The chord line is simply a straight line connecting the leading edge to the trailing edge of the airfoil whose length is defined as the chord length. The maximum thickness $t$ is located at $30 \%$ of the chord for NACA 4-series airfoil sections. The maximum camber $m$, or maximum ordinate of the mean camber line, is located a distance $p$ from the leading edge of the airfoil. The values of $m, p$, and $t$ are expressed as percentages of the chord length and represent the four digits defining the NACA 4series airfoil and are the parameters used in the optimization.

Reference [15] introduced the equations used to define the shape of a NACA 4-series airfoil. The mean camber line of the airfoil was described as an analytically defined curve which was the combination of two parabolic arcs that are tangent at the point of maximum camber. For an $x$ coordinate, the ordinate of the mean camber line can be expressed as

$$
y_{c}=\left\{\begin{array}{l}
\frac{m}{p^{2}}\left(2 p x-x^{2}\right) \\
\text { forward of maximum ordinate, } \\
\frac{m}{(1-p)^{2}}\left[(1-2 p)+2 p x-x^{2}\right], \\
\text { aft of maximum ordinate }
\end{array}\right.
$$

where $m$ is the maximum camber and $p$ is the chordwise location of the maximum camber. Once the camber line has 


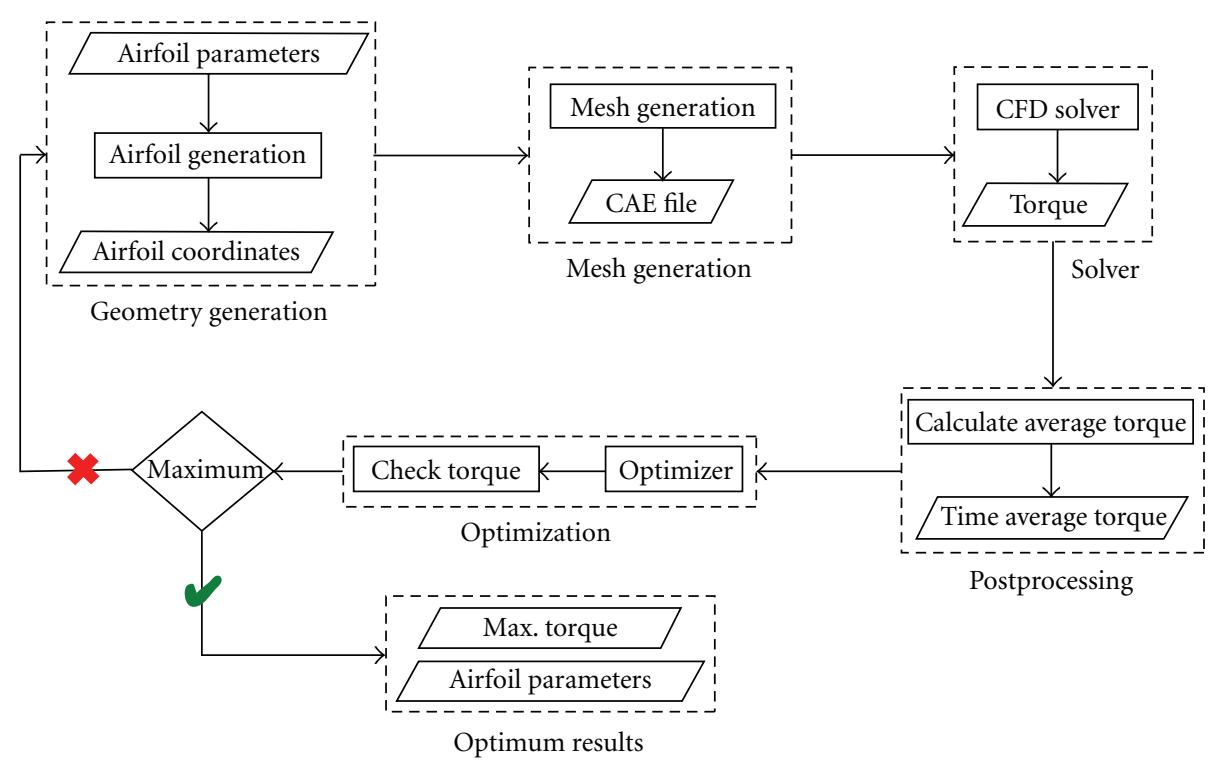

FIgURE 2: VAWT optimization methodology.

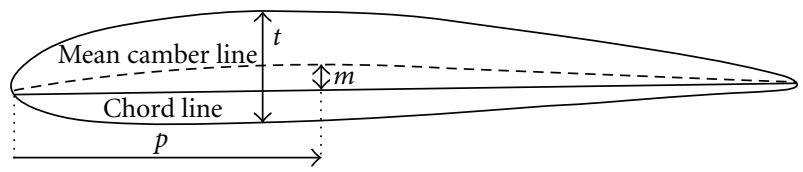

FIgURe 3: NACA 4424 4-series airfoil.

been defined, the thickness distribution can be found by the following equation:

$$
\begin{gathered}
\pm y_{t}=\frac{t}{0.20}\left(0.29690 \sqrt{x}-0.12600 x-0.35160 x^{2}\right. \\
\left.+0.28430 x^{3}-0.10150 x^{4}\right)
\end{gathered}
$$

where $t$ is the maximum thickness of the airfoil located at $30 \%$ of the chord. After the camber and thickness distribution have been defined for various $x$ locations (typically ranging from 0 to 1 ), the coordinates of the upper and lower airfoil surfaces can be obtained.

4.1.2. Airfoil Constraints. For the purposes of maintaining high cell quality during the grid generation process and a converged CFD solution, constraints were placed on the parameters defining the airfoils that could be generated for the optimization and were normalized from 0 to 1 for the optimization. The idea was to avoid airfoil geometries with large leading and trailing edge camber leading to local boundary layer cell collisions during the grid generation procedure. While placing constraints on the airfoils to be generated leads to a smaller solution space, it was done so after numerous tests to ensure that optimized geometries were found in the solution space and not on the boundaries. While this may leads one to believe that a smaller solution space leads to less feasible designs, because the optimization algorithm chosen used floating-point values to define the parameters, essentially an infinite number of airfoil designs were attainable.

4.2. Grid Generation. After the airfoil geometry for the VAWT had been defined, the next step was to discretize the computational domain as a preprocessing step in the CFD process. The act of discretizing the domain is termed grid generation and is one of the most important steps in the CFD process. For simple geometries where the direction of the flow is known beforehand, creating the grid is usually straightforward. For flows such as this, highquality structured grids can be used that can accurately capture the flow physics. However, as geometry becomes complex and the flows more difficult to predict with the onset of turbulence and separation, grid generation is no longer a trivial task. For flows such as this, unstructured grids consisting of triangles and tetrahedra provide increased flexibility and are often used.

4.2.1. Grid Considerations. Because either structured or unstructured techniques can be used to discretize a computational domain, it is important to exercise the capabilities of the solver to determine its sensitivity to the varying cell types. A good rule of thumb proposed by [7] can be seen in Figure 4. From the figure, a multiblock structured grid is said to provide the highest level of viscous accuracy; yet, it also suggests that a hybrid grid topology would provide a balanced level of accuracy and automation, an important characteristic for the optimization process.

Before a final decision was to be made on the type of grid used for the VAWT simulation, a comprehensive grid dependency study was conducted to find a grid independent solution. For this work, a family of multiblock structured grids were created alongside a family of equivalent hybrid grids. The torque was calculated for each of the grids, and a grid-independent solution was found. Details regarding the 


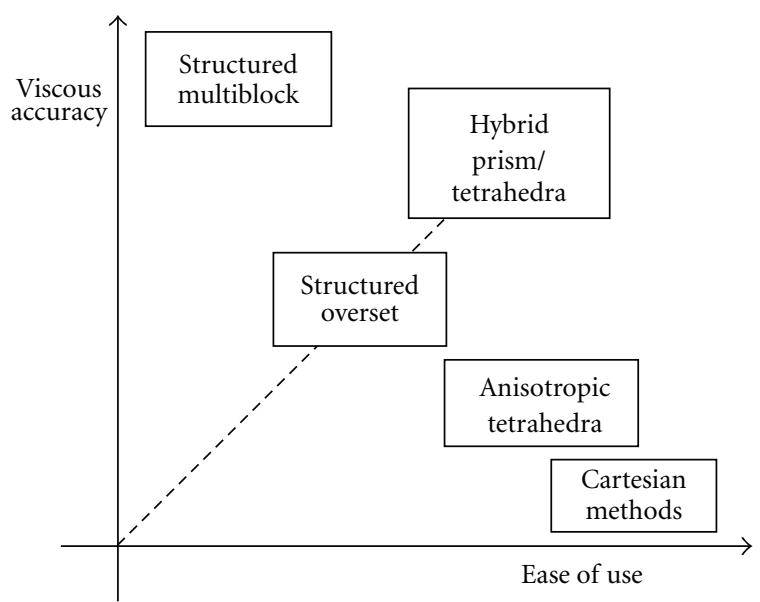

Figure 4: Grid-type accuracy versus ease of use [7].

grid generation and dependency studies will be discussed further in Section 5.

As a result of the grid dependency study and the extensive work done in the areas of aerodynamic design and optimization using unstructured grid generation techniques [16-19], a hybrid grid was chosen as the most appropriate for this work. The hybrid grid consists of a structured boundary layer transitioning to isotropic triangles in the far field and can be seen for the leading edge of a VAWT blade in Figure 5. This choice provided a flexible and completely automated approach to the grid generation of numerous VAWT geometries.

4.2.2. Pointwise. Pointwise V16.02 was used to generate the grids used for the VAWT study. For the grid dependency study, structured grids were constructed manually, while automated grid generation techniques were used to construct the hybrid grids. The far field was split into a rotating and nonrotating zone and was discretized using either structured or unstructured elements. The scripting capabilities of Pointwise were exercised while constructing the hybrid grids and allowed for the grid generation of the airfoil geometries to easily be integrated into the optimization process. A Pointwise script was written in Glyph2, based on Tcl, that imported the airfoil geometry as a list of $x, y$ coordinates and constructed the hybrid grid based on several user-defined parameters such as the initial cell height in the boundary layer and the solidity of the wind turbine. The automated script also set up the appropriate boundary conditions and exported the file for the solver.

4.3. Solver. Once the computational domain had been discretized, the equations governing fluid flow were solved using an appropriate discretization technique in order to calculate the torque. The commercial solver FLUENT v6.3 was used for this work [20]. FLUENT uses the finite volume method to discretize the integral form of the governing equations.

4.3.1. Numerical Method. For the simulation, a pressurebased segregated solver was chosen where the SIMPLE

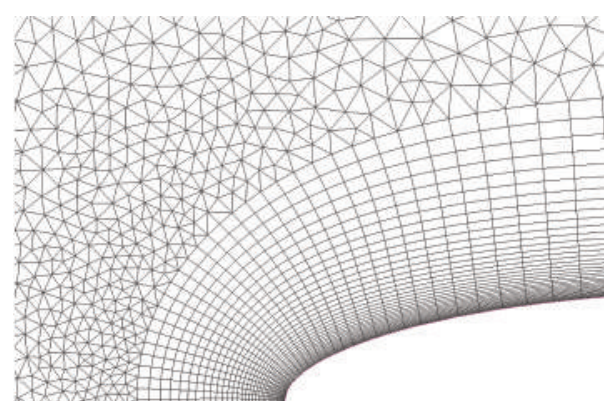

FIGURE 5: Leading edge boundary layer grid for blade geometry.

algorithm was used to handle the pressure-velocity coupling that exists. A 2nd-order interpolation scheme for pressure was used along with a 2nd-order upwind discretization scheme for the momentum equation and modified turbulent viscosity. The gradients required for the discretization of the convective and diffusive fluxes were computed using a cell-based approach. Because the simulation was time dependent, a 2nd-order implicit time integration was chosen for the temporal discretization. A time step was chosen small enough to reduce the number of iterations per time step and to properly model the transient phenomena.

Turbulence modeling was accomplished through the use of the Spalart-Allmaras one-equation turbulence model where a transport equation is solved for the eddy viscosity [21]. The $y^{+}$for the blades varied at different azimuthal locations, but consistently placed the first cell centroid of the wall-adjacent cells inside the viscous sublayer $\left(y^{+}<5\right)$ of the boundary layer. Therefore, because the grid was fine enough to resolve the viscous sublayer, the laminar stressstrain relationship $u^{+}=y^{+}$was used to determine the wall shear stress.

The system of equations resulting from the discretization and linearization of the governing integral equations were solved using an algebraic multigrid (AMG) method coupled with a point implicit Gauss-Seidel solver [22]. Due to the size and unsteady nature of the problem, the overall average computation time to achieve a quasisteady state took approximately 2.5 hours on a $2.83 \mathrm{GHz}$ Intel Core2Quad processor.

4.3.2. Computational Domain and Boundary Conditions. The interior domain containing the wind turbine blades was considered as a moving mesh, while the outer domain was stationary. The interior sliding domain rotated with a given rotational velocity for a specified $\lambda$. The inlet to the computational domain was defined as a velocity inlet with a uniform velocity component and a modified turbulent viscosity $\widetilde{v}$ equal to $5 v$, where $v$ is the molecular kinematic viscosity of air. The outlet was marked as a pressure outlet with the gauge pressure set to zero.

4.4. Postprocessing. Once the solution had been calculated using FLUENT and all relevant data had been written to a file, the average torque could then be determined. A small script parsed through the output file and saved only the 
torque values that were recorded every 15 time steps. This file then contained torque as a function of time. A graph of the torque versus time can be seen in Figure 6. At a certain point, the flow became quasi-steady, and the oscillations were more uniform. One single rotation of the wind turbine has been outlined in the figure. The three peaks in the torque represent the times at which each blade passed around the front of the wind turbine, while the three valleys represent the times at which each blade moved around the back of the wind turbine. Therefore, more blades would result in a higher frequency oscillation for the same rotation speed. In order to calculate a single scalar value of the torque for the optimizer, the oscillating torque was averaged.

4.4.1. Average Torque. In Section 2, the tangential force component driving the wind turbine, and also used to compute the torque, was a function of azimuthal location. During the simulation process, the torque was recorded as a function of time; therefore, it is important to introduce the average value of a function $f(t)$ over the interval $[a, b]$ as

$$
f_{\text {avg }}=\frac{1}{b-a} \int_{a}^{b} f(t) d t,
$$

where $a$ would represent the time at the beginning of a single rotation and $b$ is the time at the end of a single rotation. This equation states that the average value of the function $f(t)$ is equal to the integral of that function for a single rotation divided by the time required to complete a single rotation. Using the trapezoidal rule, the definite integral can be expressed by

$$
\int_{a}^{b} f(t) d t=\frac{b-a}{2 n}\left[f\left(t_{o}\right)+2 \sum_{i=1}^{n-1} f\left(t_{i}\right)+f\left(t_{n}\right)\right],
$$

where $n$ is the number of segments used to split the interval of integration. Using (11) and (12) and replacing $n$ by $\left(t_{n}-\right.$ $\left.t_{o}\right) / \Delta t$, the average value of the torque for a single rotation of the wind turbine is defined as

$$
\tau_{\text {avg }}=\frac{\Delta t}{2\left(t_{n}-t_{o}\right)}\left[\tau\left(t_{o}\right)+2 \sum_{i=1}^{n-1} \tau\left(t_{i}\right)+\tau\left(t_{n}\right)\right],
$$

where $t_{o}$ is the time at the beginning of a rotation, $t_{n}$ is the time at the end of the rotation, and $\Delta t$ is the time step used when recording the torque in FLUENT. Using (13), the average torque for the final rotation of the wind turbine was calculated and used as the objective function value driving the optimization algorithm.

4.5. Optimization. In order to maximize the average torque of the wind turbine given the NACA 4-series airfoil design parameters (Section 4.1) and the solidity and tip speed ratio design constraints, a simple and robust optimization algorithm was required. This act of searching for the minimum or maximum value of a function while varying the parameters, or values of that function, and incorporating any constraints is called optimization. In optimization, the function is often

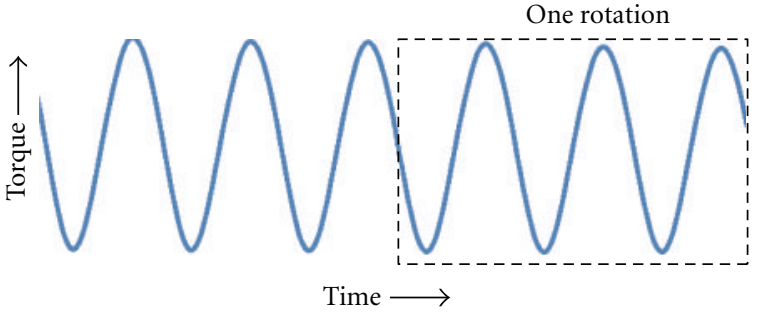

FIgURE 6: Variation of torque as a function of time.

termed the objective function or cost function, and it is the goal of the optimization algorithm to find the true minimum or maximum of that objective function as efficiently as possible. However, in design the objective function may be a rather complex, nonlinear, or nondifferentiable function that is under the influence of many parameters and design constraints. This possibility rules out any simple, gradientbased optimization algorithms such as the method of steepest descent or Newton's method, as these algorithms require the objective function to be differentiable and are only efficient at finding local minimum or maximum values. Therefore, global optimization algorithms are favored for design optimization.

4.5.1. Differential Evolution. The differential evolution (DE) algorithm is a global, stochastic direct search method aimed at minimizing or maximizing an objective function based on constraints that are represented by floating-point values rather than binary strings like most evolutionary algorithms [23-25]. The DE algorithm is robust, fast, simple, and easy to use as it requires very little user input. These traits lead to the choice of DE as the algorithm used in the current study.

4.5.2. Initialization. In order to determine the maximum value of the objective function, the DE algorithm starts with a randomly populated initial generation of NP D-dimensional parameter vectors, where NP is the number of parents in a population and $D$ is the number of parameters. For this work two optimizations were conducted. A 3-parameter optimization $(D=3)$ for a fixed tip speed ratio and solidity, as well as, a 4-parameter optimization $(D=4)$ where the solidity became a parameter, providing complete geometric flexibility. For both cases NP $=14$.

4.5.3. Mutation. After initializing the population, each target vector $\vec{x}_{i, G}$ in that generation undergoes a mutation operation given by

$$
\vec{v}_{i, G+1}=\vec{x}_{r 1, G}+F\left(\vec{x}_{r 2, G}-\vec{x}_{r 3, G}\right),
$$

where the index $r$ represents a random population member in the current generation, $F$ is a scaling factor $\in[0,2]$ dictating the amplification of the difference vector $\left(\vec{x}_{r 2, G}-\right.$ $\vec{x}_{r 3, G}$ ), and the result $\vec{v}_{i, G+1}$ is called the mutant vector. A scaling factor $F=0.8$ was selected for this work. This mutant operation is a characteristic of a variant of $\mathrm{DE}$ that utilizes a single difference operation; therefore, $\mathrm{NP} \geq 4$ such that 
the index $i$ is different than the randomly chosen values of $r 1, r 2, r 3$.

Other variants of DE exist that utilize more difference operations to determine the mutant vector. The DE strategy used in this work DE/best/2/exp utilizes two difference vectors. The idea is that by using two difference vectors the diversity of large populations can be improved, increasing the possibility that members of a population span the entire solution space and reducing the risk of premature convergence. The mutation operation for the $\mathrm{DE}$ variant used in this work is given by

$$
\vec{v}_{i, G+1}=\vec{x}_{\mathrm{best}, G}+F\left(\vec{x}_{r 1, G}+\vec{x}_{r 2, G}-\vec{x}_{r 3, G}-\vec{x}_{r 4, G}\right),
$$

where $\vec{x}_{\text {best }, G}$ represents the best performing parameter vector from the current population. This is different than the previous strategy that utilized a random population member to perform the mutation operation. The hope is that by using the best parameter vector in the population, the number of generations required for convergence will decrease.

4.5.4. Crossover. The crossover operation generates a trial vector by selecting pieces of the target vector and mutant vector. The trial vector is determined by

$$
\vec{u}_{j i, G+1}= \begin{cases}\vec{v}_{j i, G+1}, & \text { if }(\operatorname{randb}(j) \leq \mathrm{CR}) \text { or } j=\operatorname{rnbr}(\mathrm{i}), \\ \vec{x}_{j i, G}, & \text { if }(\operatorname{randb}(j)>C R) \text { and } j \neq \operatorname{rnbr}(i),\end{cases}
$$

where $\mathrm{CR}$ is the crossover constant, or crossover probability $\in[0,1]$, and $\operatorname{randb}(j)$ is a randomly chosen number $\in[0,1]$ evaluated during the $j$ th evaluation, where $j=1,2,3, \ldots, D$. If the value of $\operatorname{randb}(j)$ happens to be less than or equal to $\mathrm{CR}$, the trial vector gets populated with a parameter from the mutant vector. However, if the random number that has been generated happens to be larger than $\mathrm{CR}$, the trial vector gets a parameter from the target vector. To ensure that at least one parameter value is chosen from the mutant vector, a random value $\in 1,2,3, \ldots, D$ is chosen as $\operatorname{rnbr}(i)$. If $\mathrm{CR}=1$, all trial vector parameters will come from the mutant vector. This illustrates that the choice in CR works to control the crossover probability. In this work, $\mathrm{CR}=0.6$.

4.5.5. Selection. The last step in the DE algorithm is selection. Once the trial vector has been formed, it must be decided whether or not it should move to the next generation. Therefore, in the selection process, if the trial vector performs better than the target vector, resulting in a larger objective function value, the trial vector moves on to the next generation. However, if the newly generated trial vector is outperformed by the original target vector, the target vector remains a population member in the next generation.

In this work, the DE code generated new airfoil parameters for the first case, and new airfoil parameters and solidities for the second case of each generation through mutation, crossover, and selection operations. Each new set of parameters was used to generate the airfoils of the VAWT for which the torque could then be calculated using the FLUENT solver. The torque was then averaged by the postprocessing module and used as the objective function value driving the $\mathrm{DE}$ algorithm.

\section{Results}

The overall objective of the work was to successfully demonstrate a proof-of-concept optimization system capable of maximizing the efficiency of a three-bladed VAWT. Two test cases were conducted to demonstrate the robustness of the optimization system. The first test case was a 3parameter optimization where the both the solidity and tip speed ratio were fixed. The second test case was a 4parameter optimization for a fixed tip speed ratio. Before the final results of the optimization are presented, an overview of the grid dependency studies will be introduced. Next, the performance of a baseline geometry will be presented. Finally, the results of the two optimization test cases will be introduced and compared with the performance of the baseline geometry.

5.1. Grid Dependency Studies. For this work, a family of structured and equivalent hybrid grids were created in hope to find a grid that provided adequate resolution of the unsteady phenomena while the construction of the grid would remain highly automated for the optimization process.

To begin, a simple blade shape was used for the grid study. The VAWT consisted of three 60 degree semicircular blades with a constant thickness of $0.025 \mathrm{~m}$ giving the rotor a solidity $\sigma=1.5$. Each blade was separated by 60 degrees at a radius of $1 \mathrm{~m}$ from the axis of rotation, providing a simple geometry with which to define the initial topology. Because the blades had to spin in the simulation, an interior sliding domain was to be constructed with a radius of $25 \mathrm{~m}$. The outer stationary domain, and the extent of the far field, was defined to be $50 \mathrm{~m}$. The far field domain was large enough such that the unsteady flow characteristics would develop and dissipate inside the domain, eliminating the concern for reverse flow.

5.1.1. Structured and Hybrid Grids. Both structured and hybrid grid families were constructed for the grid dependency study. Structured grids were constructed using quadrilateral elements; therefore, opposing grid lines must contain the same number of points to construct a domain consisting of purely quadrilateral cells. A characteristic, and even a disadvantage of the structured grid topology, is that the local blade resolution used to resolve the boundary layer is propagated into the far field. This tends to lead to larger cell counts when using structured grids.

The hybrid grid topology used for this study consisted of a structured boundary layer transitioning to unstructured triangles. Unlike the structured grid, the hybrid grid topology was easy to construct and automate. The boundary layer was constructed using a normal hyperbolic extrusion technique in Pointwise [26]. The user need only to specify the initial cell height, growth rate, and the number of layers 

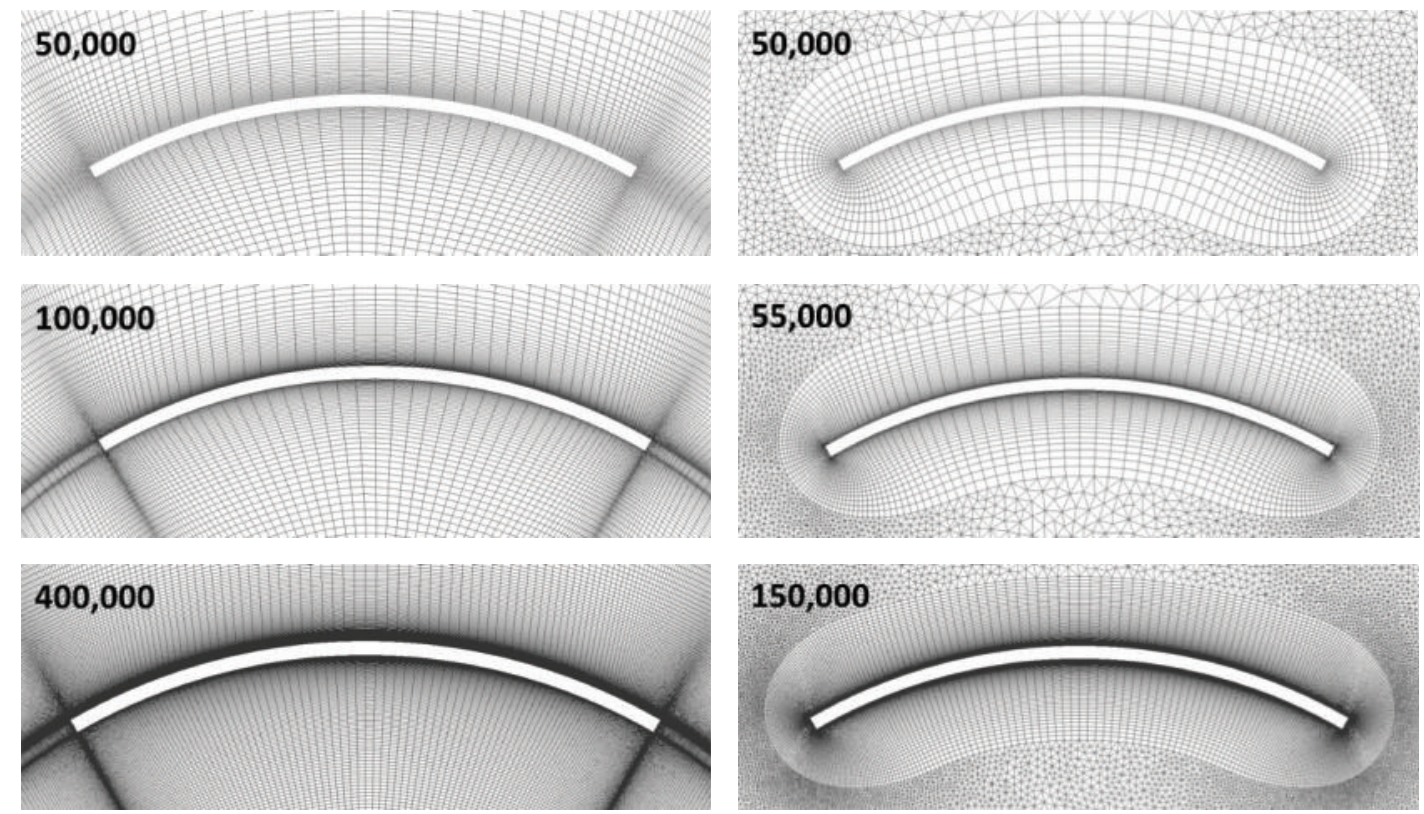

FIGURE 7: Structured and hybrid grid family comparison.

for the extrusion. A benefit from using the hybrid topology was that appropriate boundary layer resolution was obtained while maintaining a low cell count in the far field. Unlike the structured grids, the far field and boundary layer were almost entirely decoupled, resulting in a much lower cell count.

A comparison of the structured and hybrid grid families can be seen in Figure 7. The local blade resolution was preserved using the normal hyperbolic extrusion technique during the construction of the hybrid grids. As was mentioned earlier, the resolution and spacing constraints placed on the structured grid near the blade can be seen propagating away from the blade itself, whereas for the hybrid case there is a clear boundary that separates the boundary layer grid from the rest of the far field. For instance, while the coarse grids contain 50,000 cells for the same local blade resolution, once the spacing was adjusted to achieve higher resolution, the structured grid contained 100,000 cells as opposed to the hybrid grid containing only 55,000 cells. In this case, the local blade resolution that was enforced for the structured grid propagated into the far field. However, while the local blade resolution changed for the hybrid grid, the far field remained unaffected.

5.1.2. Grid-Independent Solution. The torque was calculated for each grid using the FLUENT solver, the settings of which were discussed in Section 4. A time step of $\Delta t=2 \pi / \omega N$ was used where $\omega$ is the rotation rate, and $N$ is the number of cells in the circumferential direction. This was calculated for the 100,000 cell structured mesh with $\omega=10 \mathrm{rad} / \mathrm{s}$ to be approximately $0.001 \mathrm{~s}$, the time step that was used for all simulations. This represents the amount of time for the sliding domain to move one grid point in the circumferential direction and was found adequate for the simulation.
Convergence was monitored by observing the residuals as well as the torque. In the ideal case, the residuals should converge to true zero. However, a more relaxed convergence criteria of $1 e-5$ was enforced for continuity, momentum, and modified turbulent viscosity. The residuals were monitored every time step, while the torque was recorded every 15 time steps. The solution consistently became quasi-steady after 5 rotations, approximately 3150 time steps. The time step used for the simulation allowed the solution to converge after 30 iterations per time step, resulting in nearly 100,000 iterations to achieve a quasi-steady state.

The average torque was calculated for the last rotation of the wind turbine for each of the grids. From this grid dependency study, it was found that the 100,000 cell structured grid and 55,000 cell hybrid grid seem to exhibit grid convergence. However, due to the complexity of the topology required to construct the structured grid and the difficulty of applying this topology to varying geometries, the 55,000 cell hybrid grid topology was chosen for the optimization.

To demonstrate the automation and quality of the hybrid grid topology for arbitrary blade geometries, a hybrid grid was constructed for a high-solidity VAWT geometry and a low-solidity geometry seen in Figure 8 . The normal hyperbolic extrusion created layers of quads that marched smoothly away from the blade, transitioning to isotropic triangles. Utilizing this technique resulted in high-quality, automated hybrid grid generation for all airfoil geometries analyzed throughout the optimization process.

5.2. Baseline Geometry. A baseline VAWT geometry was selected with which the results of the optimization could be compared. The idea was to select a typical VAWT airfoil cross-section. Therefore, the NACA 0015 was selected as the 


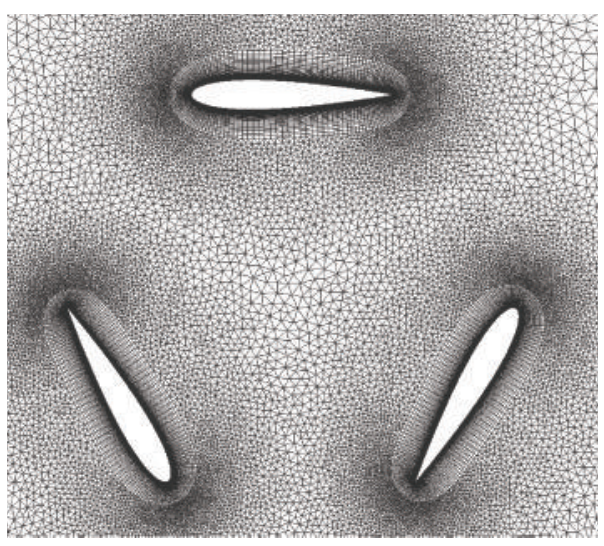

(a)

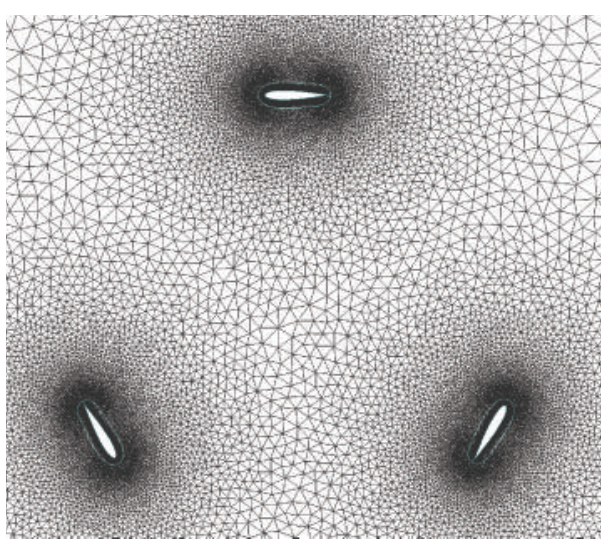

(b)

FIGURE 8: Hybrid grids for VAWT geometry, $\sigma=1.5$ (a) and $\sigma=0.4$ (b).

baseline airfoil cross-section simply due to the fact that a number of researchers attribute this geometry with good overall aerodynamic performance [3-5]. The NACA 0015 airfoil cross-section can be seen in Figure 9.

5.2.1. Baseline Performance. The performance of the baseline three-bladed VAWT utilizing NACA 0015 airfoil crosssections was evaluated for $\sigma=1.5$ and $\lambda=[0.5,1.5]$. By keeping $\omega$ constant at $10 \mathrm{rad} / \mathrm{s}$ for $\sigma=1.5, V_{\infty}$ was adjusted to control $\lambda$. This provided relevant performance data surrounding $\lambda=1$, the tip speed ratio design constraint for the optimization. A total of 5 simulations were run to build up a performance envelope for the baseline geometry. The average torque was calculated for each simulation and was used to determine the coefficient of performance. The results of the analysis can be seen in Figure 10.

Figure 10 defines the performance envelope for a VAWT utilizing the NACA 0015 airfoil for $\sigma=1.5$. It can be seen that there exists a point at which the efficiency is highest $(\lambda \approx 1.2)$ and can be described as the optimum tip speed ratio for the geometry. As expected, as the wind speed changes, driving the tip speed ratio away from the optimum, the efficiency decreases. From this figure, it can be deduced that, in order for the baseline wind turbine design to perform optimally at $\lambda=1$, the solidity of the rotor would have to be changed while retaining the NACA 0015 airfoil cross-section. This would give the wind turbine even more geometric flexibility and lead to the decision to allow the solidity to become a design parameter in the 4-parameter optimization test case. However, in order to compare the results of the NACA 0015 with the optimization test cases and demonstrate how VAWT design can benefit from using optimization, the solidity of the baseline geometry was not adjusted.

5.3. Case 1: 3-Parameter Optimization. The first test case to run through the optimization system was the 3-parameter case. The idea was to maximize the torque of the VAWT for a fixed solidity and tip speed ratio. The case ran for approximately 1 week on the cluster described in Section 4,

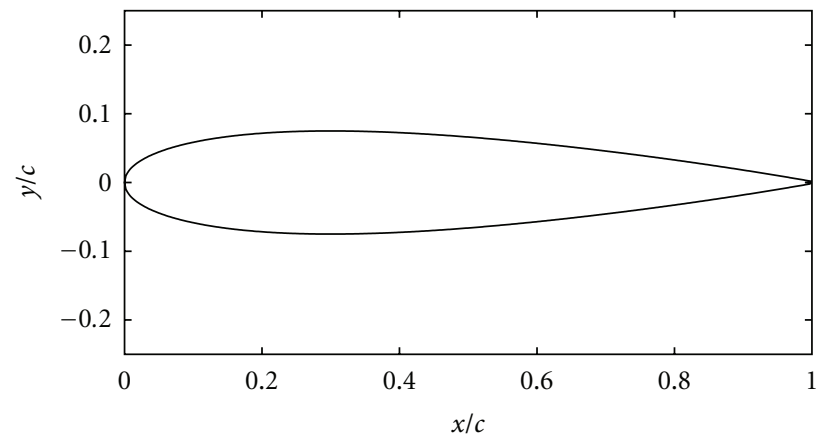

FiguRE 9: NACA 0015 baseline geometry.

after which the maximum number of user specified generations was reached $(G=11)$. Because there was no guarantee that the optimization algorithm would find the optimum design, the goal was to obtain an improved design that was able to achieve a higher efficiency than the baseline geometry.

To demonstrate the capabilities of the optimization system and show that 1 week was enough time to achieve an optimized geometry, 2 unique initial populations that were randomly generated by the $\mathrm{DE}$ code were run through the optimization system. The results of the 2 unique runs will be presented and compared with the baseline geometry. The optimization is said to have been successful if the VAWT utilizing the optimized airfoil cross-section achieved a higher efficiency than the baseline geometry at the design tip speed ratio $(\lambda=1)$.

5.3.1. Optimization Results. Due to the nature of the DE algorithm, the initial population is random and completely different for the 2 runs conducted. The reason for starting with 2 different initial populations was to ensure that 11 generations was a sufficient amount time to find an optimized design while avoiding premature convergence or stagnation.

The diversity of the population for all generations can seen in Figure 11. This figure illustrates how the COP varies with generation. NACAopt-RUN1 and NACAoptRUN2 refer to the 2 unique runs conducted for the 1 st 


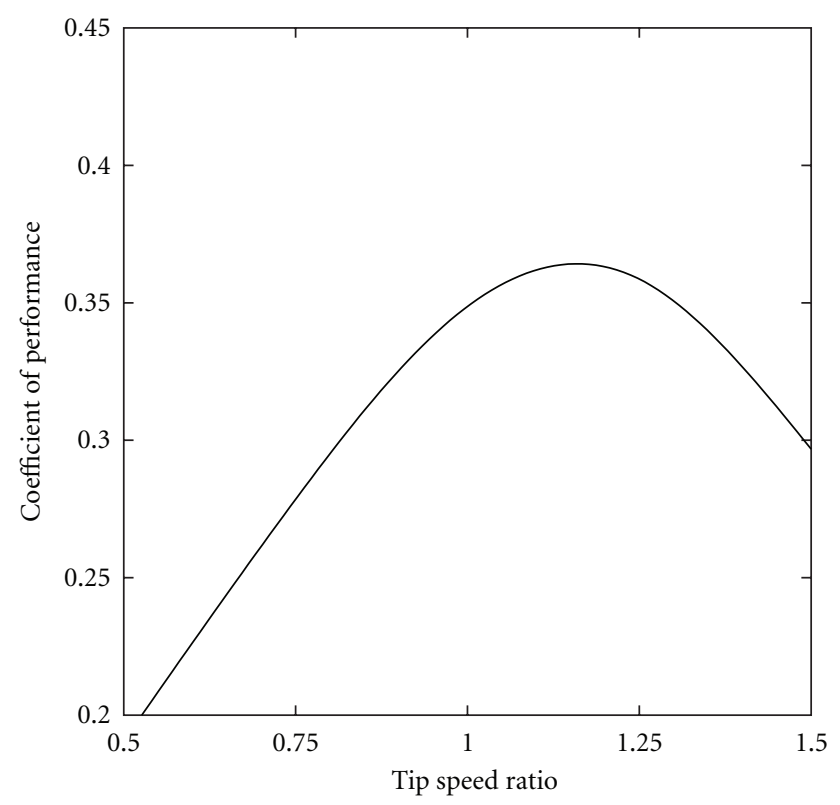

Figure 10: NACA 0015 performance envelope, $\sigma=1.5$.

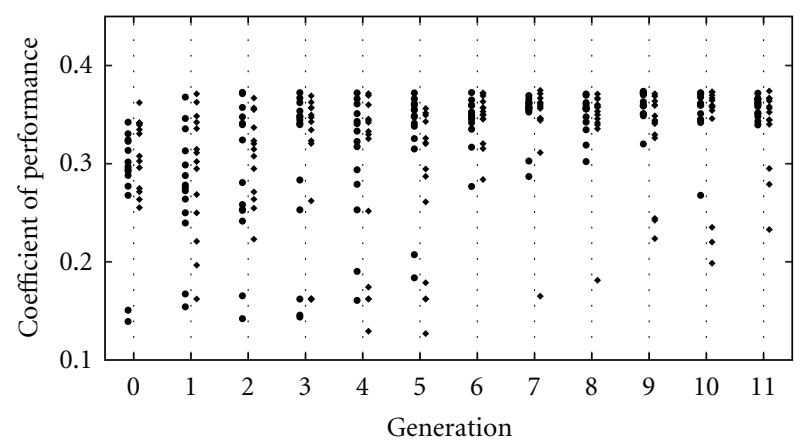

- NACAopt-RUN1

- NACAopt-RUN2

FIGURE 11: COP versus generation for all population members, 1 st test case.

test case. It can be seen that the populations for each of the first 5 generations are quite diverse. However, after the 5th generation the populations begin to converge while still remaining somewhat diverse, a characteristic of the stochastic nature of the DE algorithm.

While Figure 11 illustrates the diversity of the population for each generation, Figure 12 provides a history of the best overall objective function value throughout the optimization. If the COP at the current generation happens to be higher than the previous maximum, it is replaced and the new airfoil design parameters are used to generate the next population. After 11 generations NACAopt-RUN1 and NACAopt-RUN2 were able to achieve a maximum COP of 0.373 and 0.374 , respectively, despite the fact that each run was initialized from a different initial population.

The optimized geometry for both runs can be seen in Figure 13 compared with the NACA 0015 cross-section. The NACAopt-RUN1 airfoil has a maximum camber of $0.0094 c$, a maximum camber location of $0.599 c$, and a maximum

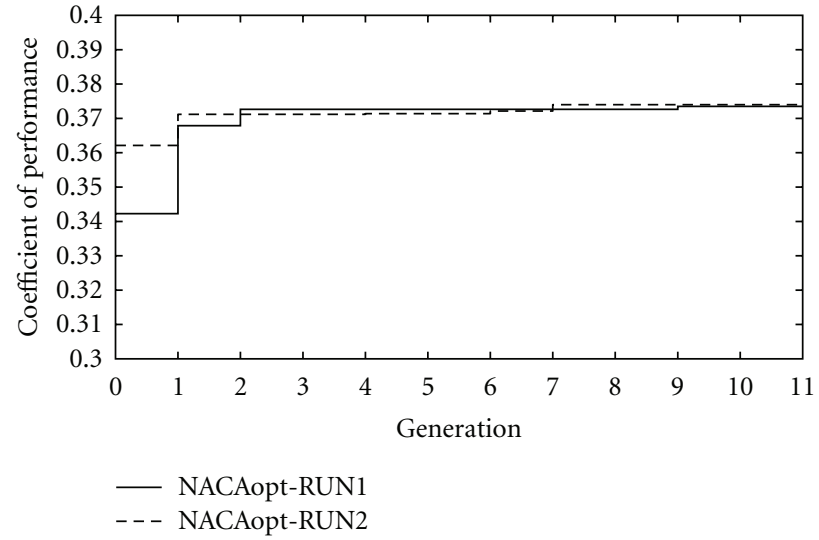

Figure 12: Max COP versus generation, 1st test case.

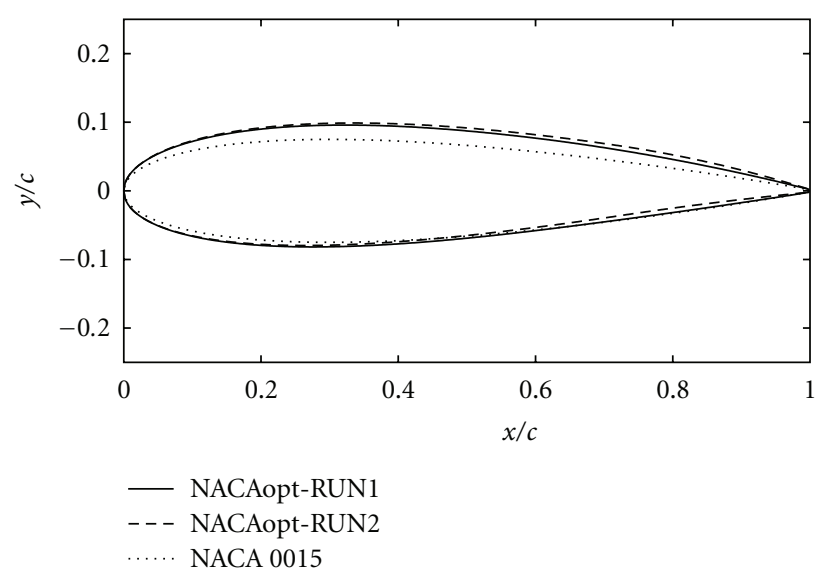

FIGURE 13: Optimized NACA 4-series airfoil geometry, 1st test case.

thickness of $0.177 c$, where $c$ is the chord length of the airfoil. The choice in a cambered airfoil geometry over a symmetric cross-section could be an indication that slight camber increases the efficiency of high-solidity rotors that experience undesirable blade vortex interactions. The fact that the maximum COP and the optimized airfoil crosssection for both runs are indistinguishable indicates that 11 generations is sufficient. Therefore, it is unnecessary to discuss the performance of both VAWTs and only the performance of the NACAopt-RUN1 will be presented.

The performance envelope for the VAWT using the NACAopt airfoil cross-section can be seen in Figure 14. Because the optimization was run for $\lambda=1$, the blade shape was tailored to perform as best as possible at this value. Therefore, the optimum tip speed ratio is much closer to 1 , signifying that the solidity of the rotor would have to be adjusted to achieve maximum efficiency at $\lambda=1$.

5.3.2. Baseline Comparison. While the optimization algorithm was able to find an optimized NACA 4-series geometry for $\sigma=1.5$ and $\lambda=1$ with very little user input and little or no designer intuition or experience, it had to be compared with the baseline geometry to quantify the performance gained by using such approach. The performance envelopes for the NACAopt and NACA 0015 VAWT designs are shown 


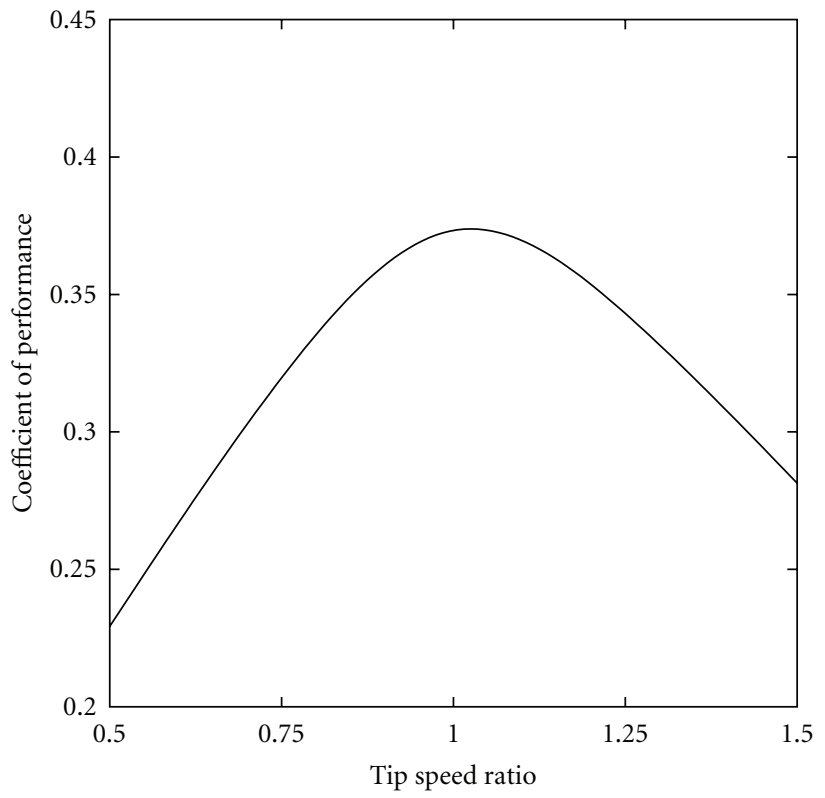

Figure 14: NACAopt performance envelope, 1st test case.

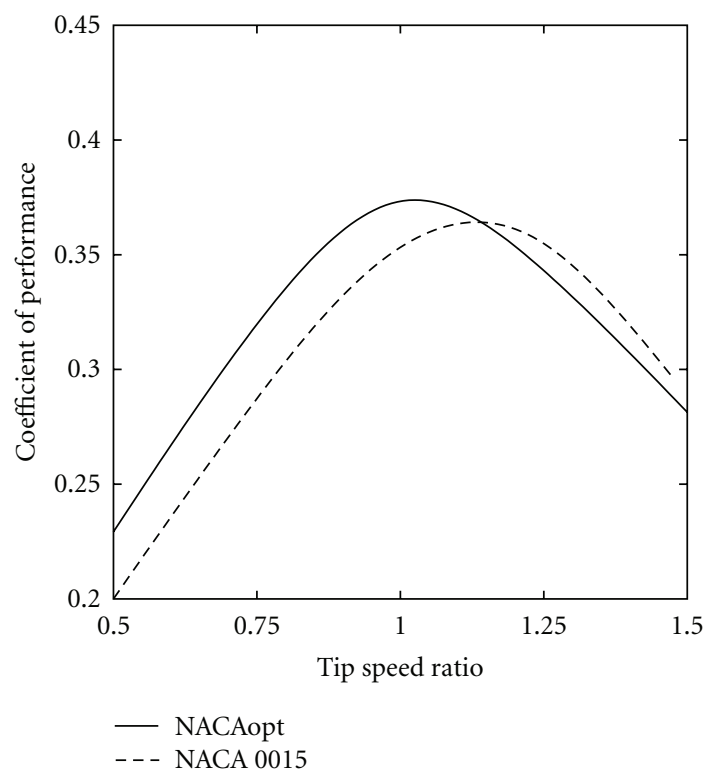

Figure 15: NACAopt versus NACA 0015 performance, 1st test case.

in Figure 15. For the design tip speed ratio $\lambda=1$, the NACAopt design has a COP $=0.373,2.4 \%$ higher than the NACA 0015 baseline geometry, which over the lifetime of the VAWT is considered a significant improvement.

In order to understand the mechanism for improved efficiency over the baseline geometry, the torque for a single rotation was observed, seen in Figure 16. While the frequency of the oscillation in the torque is the same for both geometries, the peak-to-peak amplitude for the NACA 0015 is higher than that of the NACAopt geometry. The higher thickness of the NACAopt geometry allows for such a crosssection to achieve a slightly higher angle of attack before stall than that of the NACA 0015 airfoil. Therefore, due to

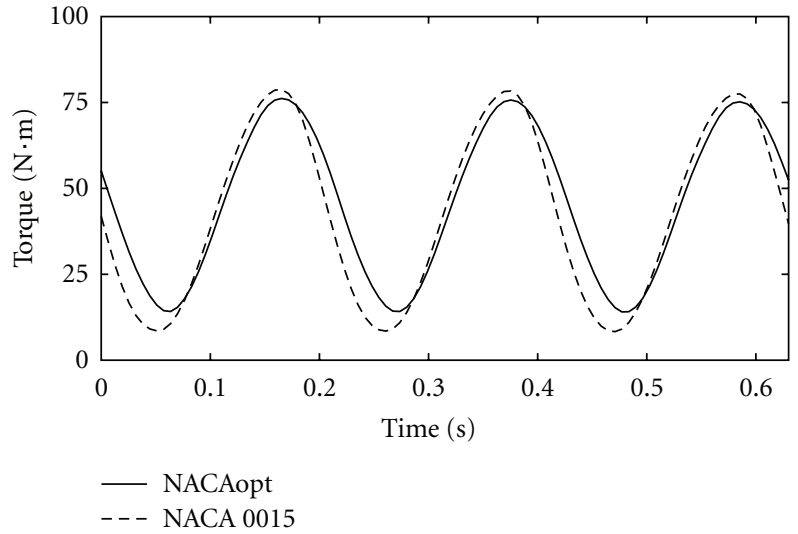

FIgURE 16: NACAopt versus NACA 0015 torque for $\lambda=1$, 1st test case.

the increase in drag associated with the dynamic stall of the NACA 0015, a higher cyclic loading is observed. Not only did the NACAopt obtain a higher efficiency, but also a reduction in cyclic loading which could lead to a longer lifespan than the NACA 0015 geometry.

Interesting flow field phenomena were captured when visualizing the vorticity seen in Figure 17. The image clearly reveals that the wake of one blade actually interacts with the trailing blade, a characteristic typical of high-solidity rotors. This interaction disturbs the flow, altering the velocity field around the trailing blade, and is most likely the reason a cambered airfoil was chosen for this high-solidity geometry. In the pair of images labeled $b$, a leading edge separation bubble can be seen forming on the lower left blade for the NACA 0015 geometry. However, the same phenomena is not observed for the NACAopt geometry. In $c$ and $d$ as the blade continues to rotate counterclockwise the separation bubble becomes larger and eventually separates, contributing the trailing vortex and increasing its strength. The increase in the efficiency of the NACAopt geometry can be attributed to the airfoil cross-section's favorable characteristics at higher angles of attack, leading to the elimination of the leading edge separation bubble and a reduction in cyclic loading.

5.4. Case 2: 4-Parameter Optimization. The second test case to run through the optimization system was the 4-parameter case. For this case, the idea was to maximize the torque of the VAWT for a fixed tip speed ratio and let the solidity of the rotor become a design variable. Allowing the solidity to become a parameter gave the wind turbine complete geometric flexibility. Not only was the blade shape allowed to change, but also the size of the blade. The case ran for approximately 10 days on the cluster described in Section 4, after which the maximum number of user specified generations was reached $(G=20)$. The goal for this case was to demonstrate that, with increased geometric flexibility, a VAWT could be designed that outperformed the baseline NACA 0015 geometry, providing a solution that was beyond the intuition of the designer. Similar to the first case, the optimization was run for $\lambda=1$. 

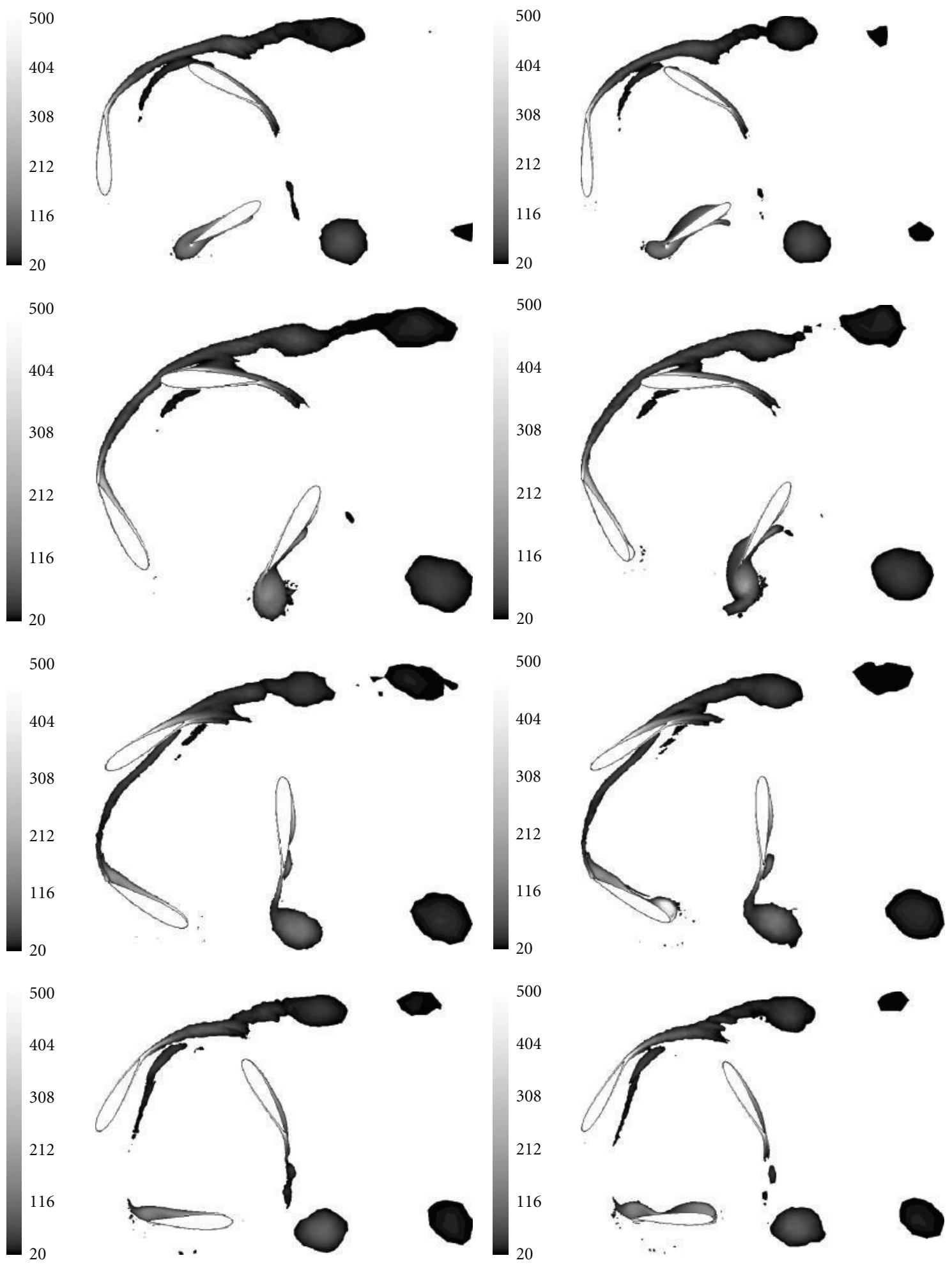

(a)

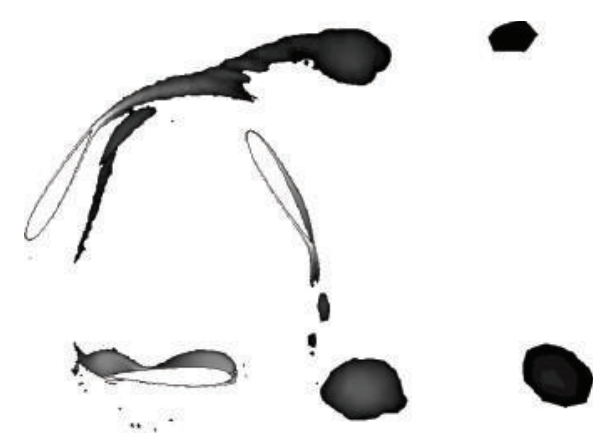

(b)

Figure 17: NACAopt (a) and NACA 0015 (b) vorticity contours, 1st test case. 
5.4.1. Optimization Results. The objective function value, COP, can be seen for each generation in Figure 18. Similar to the first case, this figure illustrates how the COP varies with generation. For the first 8 generations, the stochastic nature of the DE algorithm is evident. However, after the 8th generation the populations begin to converge and lose their diversity.

The history of the maximum COP throughout the optimization is shown in Figure 19. After just the 2nd generation, the maximum COP does not change for 9 generations, signifying the possibility of premature convergence. However, looking back at Figure 18, the population has not lost its diversity, an indication that the algorithm did not converge prematurely. After the 9th generation the maximum COP began changing every couple of generations and eventually reached a COP of 0.409 after the 20th generation.

The optimized airfoil cross-section can be seen in Figure 20. The NACAopt airfoil is symmetric with a maximum thickness of $0.237 c$ and a rotor solidity of 0.883 . The blade is $58 \%$ thicker than the NACA 0015 and the solidity has been reduced by $40 \%$. The choice in a symmetric airfoil is significant. Because low-solidity rotors do not experience strong blade vortex interactions, the positive and negative angles of attack that the blades experience are of the same magnitude; therefore, symmetric airfoils are typically used. Compared with the 3-parameter optimization this dramatic change in geometry indicates that, when given the opportunity, the optimization tends to seek out symmetric airfoil cross-sections. In the previous case, a slightly cambered geometry was chosen; this was most likely the result of the smaller design space associated with the 3parameter optimization.

The performance envelope for the NACAopt geometry can be seen in Figure 21. Allowing the solidity to become a design parameter improved the peak performance over both the baseline geometry and the 3-parameter optimization. However, contrary to initial belief, the optimum tip speed ratio is not equivalent to the design tip speed ratio. While the 4-parameter optimization allowed the entire geometry to adjust for best performance, this was accomplished only for a single tip speed ratio, the design tip speed ratio. Therefore, there was no guarantee that the optimum tip speed ratio and design tip speed ratio would coincide, a claim that has been considered a topic for future research.

5.4.2. Comparison. The optimization algorithm was able to find an optimized NACA 4-series airfoil cross-section with $\sigma=0.883$ for $\lambda=1$ in 20 generations, the performance of which was compared with the baseline NACA 0015 geometry shown in Figure 22. The NACAopt design was able to achieve a COP $=0.409$ at $\lambda=1$, ultimately resulting in a $6 \%$ increase in efficiency over the baseline NACA 0015 geometry and even a $3.6 \%$ increase in efficiency when compared with the 3parameter optimization. This case successfully demonstrated that allowing the solidity to become a parameter, and hence providing complete geometric flexibility, resulted in a significant increase in the efficiency.

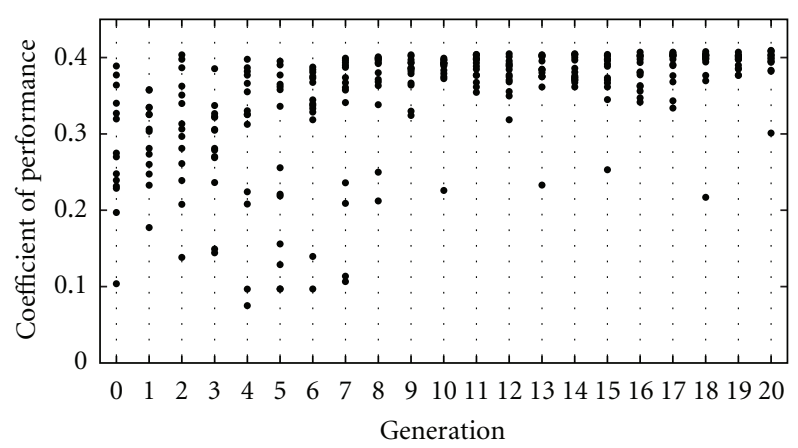

FIGURE 18: COP versus generation for all population members, 2nd test case.

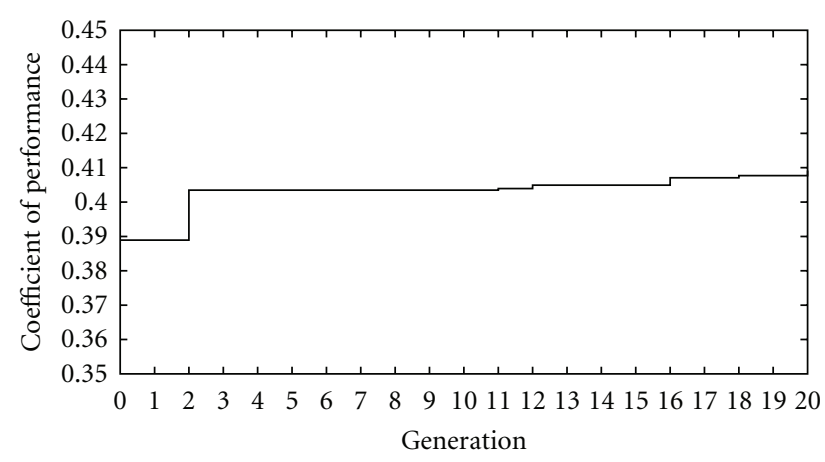

Figure 19: Max COP versus generation, 2nd test case.

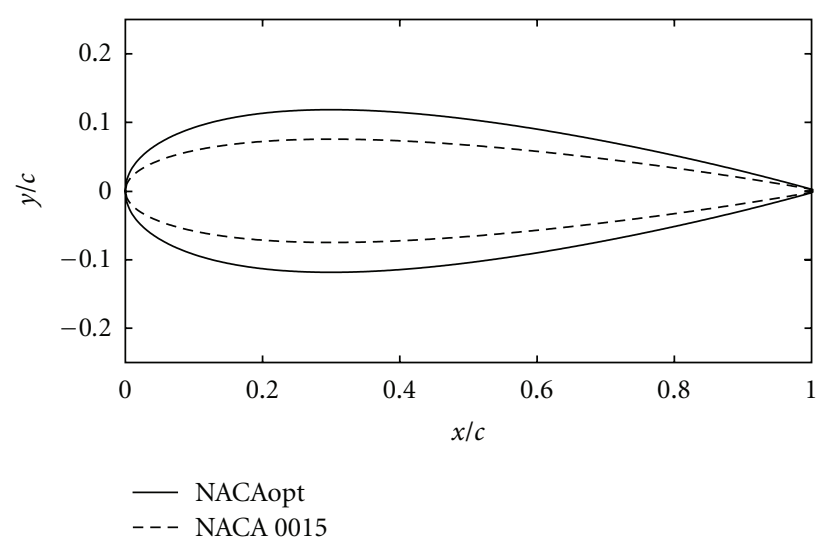

FIGURE 20: Optimized NACA 4-series airfoil geometry $(\sigma=0.883)$, 2nd test case.

In an attempt to determine the reason for the higher efficiency associated with the NACAopt geometry, the torque for a single rotation of the optimized design was compared with the baseline geometry, shown in Figure 23. While the frequency of the oscillation is the same because both designs operate at $\lambda=1$, there is an obvious phase shift in the torque oscillations resulting in the maximum performance of the NACAopt design occurring slightly earlier than the NACA 0015 rotor. The NACAopt designs $40 \%$ reduction in solidity coupled with the $58 \%$ increase in thickness allowed for such 


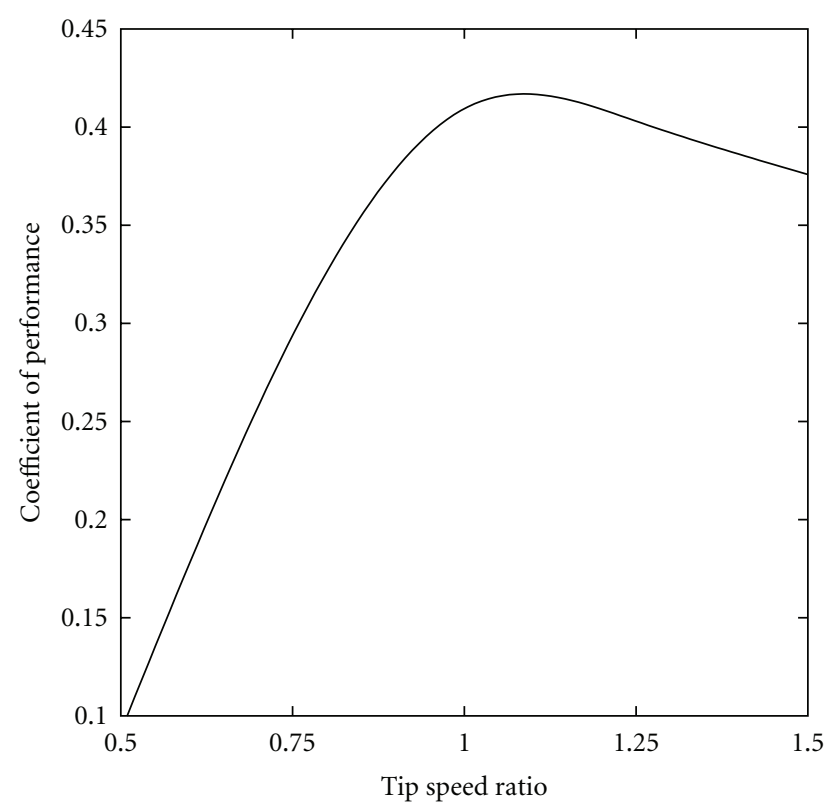

Figure 21: NACAopt performance envelope, 2nd test case.

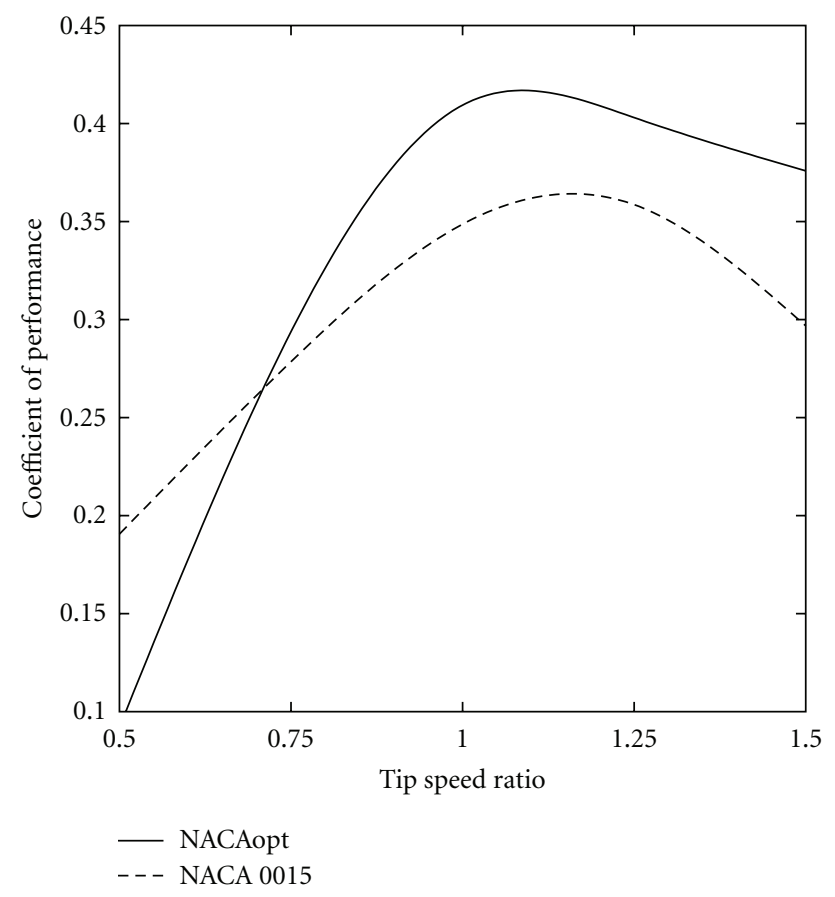

Figure 22: NACAopt versus NACA 0015 performance, 2nd test case.

a cross-section to achieve a higher overall peak performance when compared with the $15 \%$ thick NACA 0015 geometry.

\section{Conclusions}

This work successfully demonstrated a fully automated process for optimizing the airfoil cross-section of a VAWT. The generation of NACA airfoil geometries, hybrid mesh generation, and unsteady CFD were coupled with the DE

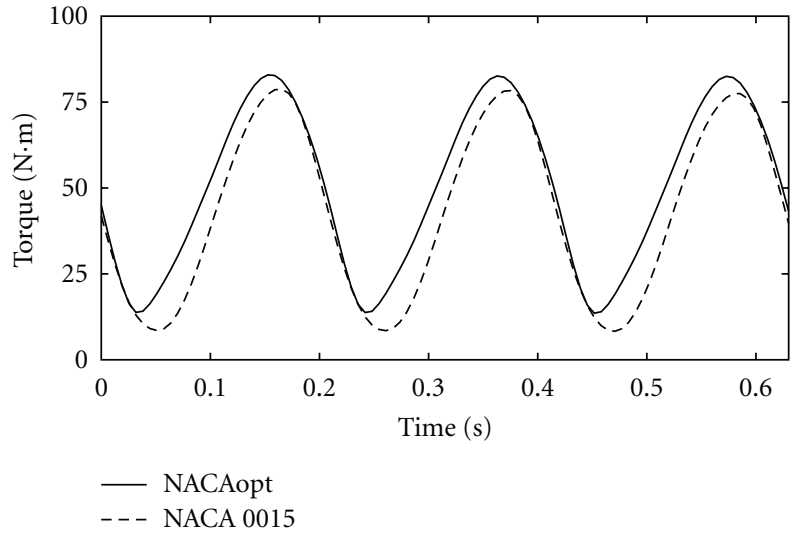

Figure 23: NACAopt versus NACA 0015 torque for $\lambda=1$, 2nd test case.

algorithm subject to tip speed ratio, solidity, and blade profile design constraints. The optimization system was then used to obtain an optimized blade cross-section for 2 test cases, resulting in designs that achieved higher efficiency than the baseline geometry. The optimized design for the 1st test case achieved an efficiency $2.4 \%$ higher than the baseline geometry. The increase in efficiency of the optimized geometry was attributed to the elimination of a leading edge separation bubble that was causing a reduction in efficiency and an increase in cyclic loading. For the 2 nd test case, the VAWT was given complete geometric flexibility as both the blade shape and rotor solidity were allowed to change during the optimization process. This resulted in a geometry that achieved an efficiency $6 \%$ higher than the baseline NACA 0015 geometry. This increase in efficiency was a result of the $40 \%$ decrease in solidity coupled with the $58 \%$ increase in thickness, leading to a slight phase shift in the torque and higher overall peak performance. While this study is significant, it represents an initial step towards the development of an operational VAWT utilizing an optimized blade crosssection and requires further research and development.

\section{References}

[1] M. Islam, D. S. K. Ting, and A. Fartaj, "Aerodynamic models for Darrieus-type straight-bladed vertical axis wind turbines," Renewable and Sustainable Energy Reviews, vol. 12, no. 4, pp. 1087-1109, 2008.

[2] I. Paraschivoiu, F. Saeed, and V. Desobry, "Prediction capabilities in vertical-axis wind turbine aerodynamics," in Proceedings of the World Wind Energy Conference and Exhibition, Berlin, Germany, 2002.

[3] C. J. Ferreira, H. Bijl, G. van Bussel, and G. van Kuik, "Simulating Dynamic Stall in a 2D VAWT: modeling strategy, verification and validation with Particle Image Velocimetry data," Journal of Physics, vol. 75, no. 1, Article ID 012023, 2007.

[4] J. C. Vassberg, A. K. Gopinath, and A. Jameson, "Revisiting the vertical-axis wind-turbine design using advanced computational fluid dynamics," in Proceedings of the 43rd AIAA Aerospace Sciences Meeting and Exhibit, pp. 12783-12805, Reno, Nev, USA, January 2005. 
[5] J. Edwards, N. Durrani, R. Howell, and N. Qin, "Wind tunnel and numerical study of a small vertical axis wind turbine," in Proceedings of the 46th AIAA Aerospace Sciences Meeting and Exhibit, Reno, Nev, USA, January 2008.

[6] M. Torresi, S. M. Camporeale, P. D. Strippoli, and G. Pascazio, "Accurate numerical simulation of a high solidity Wells turbine," Renewable Energy, vol. 33, no. 4, pp. 735-747, 2008.

[7] T. J. Baker, "Mesh generation: art or science?" Progress in Aerospace Sciences, vol. 41, no. 1, pp. 29-63, 2005.

[8] A. Ueno and B. Dennis, "Optimization of apping airfoil motion with computational uid dynamics," The International Review of Aerospace Engineering, vol. 2, no. 2, pp. 104-111, 2009.

[9] R. Bourguet, G. Martinat, G. Harran, and M. Braza, "Aerodynamic multi-criteria shape optimization ofvawt blade profile by viscous approach," Wind Energy, pp. 215-219, 2007.

[10] M. Jureczko, M. Pawlak, and A. Męzyk, "Optimisation of wind turbine blades," Journal of Materials Processing Technology, vol. 167, no. 2-3, pp. 463-471, 2005.

[11] P. Fuglsang and H. A. Madsen, "Optimization method for wind turbine rotors," Journal of Wind Engineering and Industrial Aerodynamics, vol. 80, no. 1-2, pp. 191-206, 1999.

[12] H. Rahai and H. Hefazi, "Vertical axis wind turbine with optimized blade profile," Tech. Rep. 7,393,177 B2, July 2008.

[13] D. Dellinger, "Wind - average wind speed," 2008, http://www .ncdc.noaa.gov/oa/climate/online/ccd/avgwind.html.

[14] N. S. Çetin, M. A. Yurdusev, R. Ata, and A. Özdemir, "Assessment of optimum tip speed ratio of wind turbines," Mathematical and Computational Applications, vol. 10, no. 1, pp. 147-154, 2005.

[15] I. Abbott and A. von Doenhoff, Theory of Wing Sections Including a Summary of Airfoil Data, Dover, New York, NY, USA, 1959.

[16] W. Anderson and D. Bonhaus, "Aerodynamic design on unstructured grids for turbulent ows," Tech. Rep. 112867, National Aeronautics and Space Administration Langley Research Center, Hampton, Va, USA, 1997.

[17] J. Elliott and J. Peraire, "Practical three-dimensional aerodynamic design and optimization using unstructured meshes," AIAA Journal, vol. 35, no. 9, pp. 1479-1485, 1997.

[18] M. Giles, "Aerodynamic design optimisation for complex geometries using unstructured grids," Tech. Rep. 97/08, Oxford University Computing Laboratory Numerical Analysis Group, Oxford, UK, 2000.

[19] E. J. Nielsen and W. K. Anderson, "Aerodynamic design optimization on unstructured meshes using the Navier-Stokes equations," AIAA Journal, 1998, AIAA-98-4809.

[20] Fluent, "Fluent 6.3 user's guide," Tech. Rep., Fluent Inc., Lebanon, NH, USA, 2006.

[21] P. Spalart and S. Allmaras, "A one-equation turbulence model for aerodynamic ows," in Proceedings of the 30th AerospaceSciences Meeting and Exhibit, Reno, Nev, USA, 1992, 92-0439.

[22] J. Ferziger and M. Peric, Computational Methods for Fluid Dynamics, Springer, New York, NY, USA, 3rd edition, 2002.

[23] K. V. Price, "Differential evolution: a fast and simple numerical optimizer," in Proceedings of the Biennial Conference of the North American Fuzzy Information Processing Society (NAFIPS '96), pp. 524-527, June 1996.

[24] R. Storn and K. Price, "Differential evolution-a simple and efficient adaptive scheme for global optimizationover continuous spcaes," Tech. Rep. TR-95-012, ICSI, March 1995.
[25] R. Storn and K. Price, "Differential evolution—a simple and efficient heuristic for global optimization over continuous spaces," Journal of Global Optimization, vol. 11, no. 4, pp. 341359, 1997.

[26] J. Steinbrenner, N. Wyman, and J. Chawner, "Development and implementation of gridgen's hyperbolicpde and extrusion methods," in Proceedings of the 38th AIAA Aerospace Sciences Meeting and Exhibit, Reno, Nev, USA, 2000. 

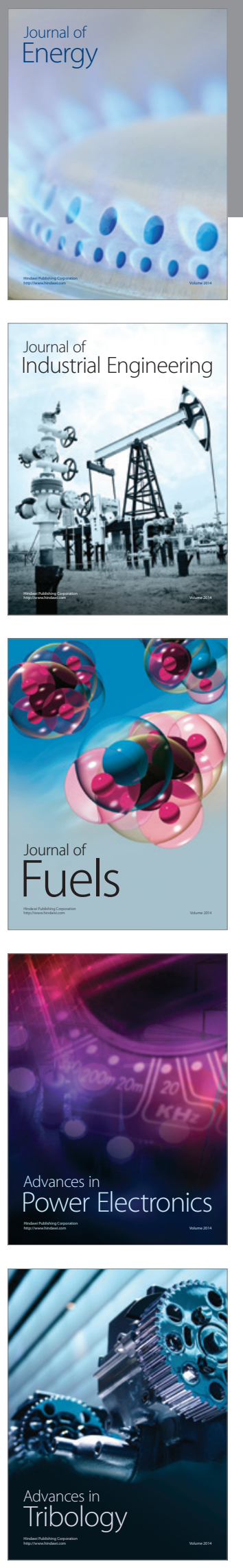
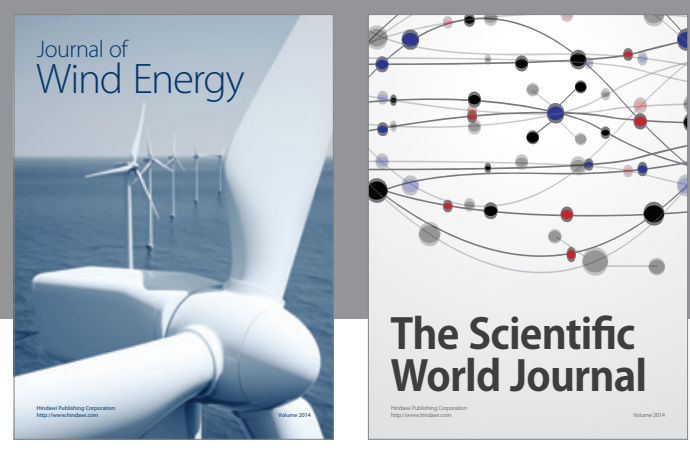

The Scientific World Journal

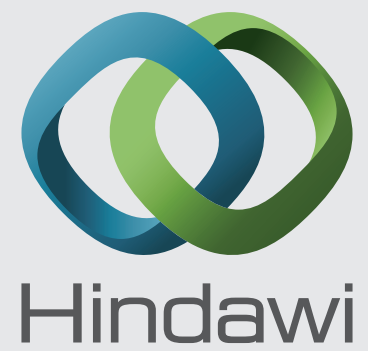

Submit your manuscripts at http://www.hindawi.com
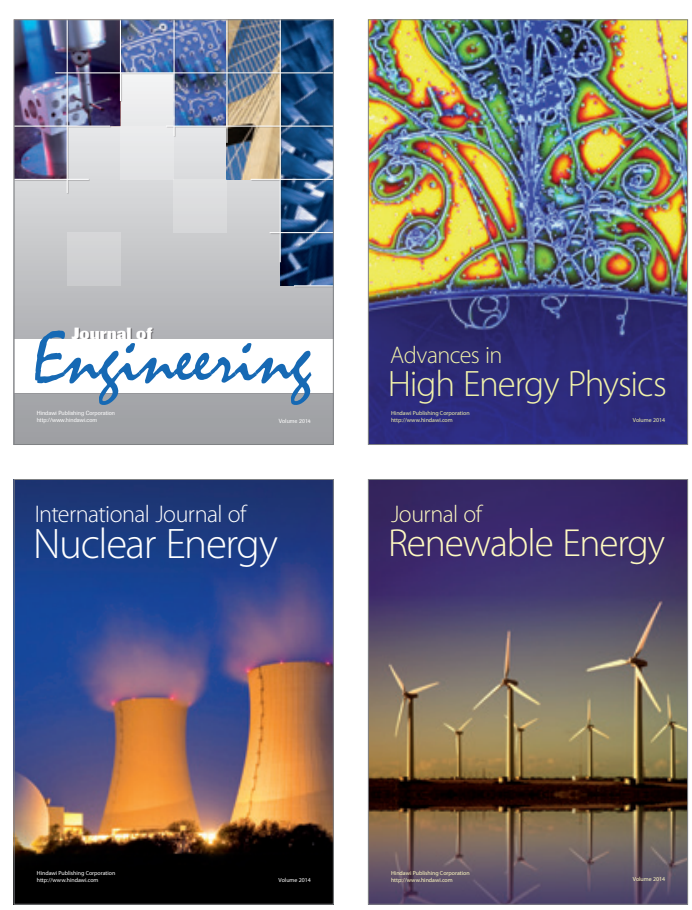

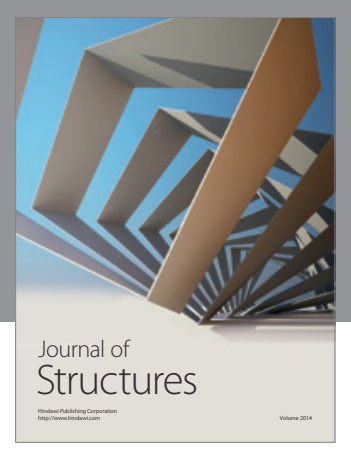

Rotating
Mechinery
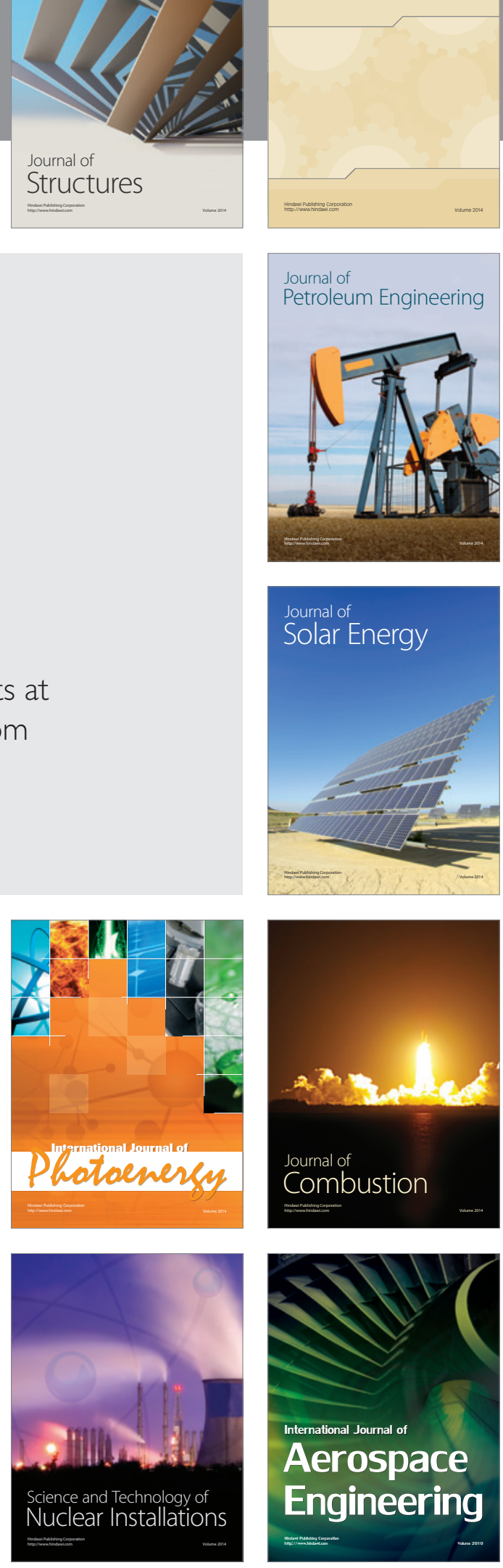